\title{
MALTRATO FÍSICO Y MALTRATO PSICOLÓGICO EN MUJERES VÍCTIMAS DE VIOLENCIA EN EL HOGAR: UN ESTUDIO COMPARATIVO
}

\author{
PEDRO J. AMOR, ENRIQUE ECHEBURÚA, PAZ DE CORRAL, \\ BELÉN SARASUA e IRENE ZUBIZARRETA \\ Universidad del País Vasco
}

(Aceptado en septiembre de 2001)

\begin{abstract}
En este trabajo se han estudiado diferentes variables sociodemográficas y psicopatológicas en una muestra de 250 víctimas de maltrato doméstico en tratamiento psicológico. Asimismo se compararon a las víctimas en función del tipo de maltrato sufrido -físico ( $\mathrm{N}=156)$ y psicológico $(\mathrm{N}=94)$ - en todas las variables estudiadas. Los resultados ponen de manifiesto la existencia de repercusiones psicopatológicas importantes en el ámbito de la ansiedad -el trastorno de estrés postraumático especialmente- y de la depresión y en el funcionamiento en la vida diaria, sin que haya diferencias significativas entre ambos grupos a pesar de la mayor gravedad de las conductas implicadas en el grupo del maltrato físico. Se comentan las implicaciones de este estudio para la práctica clínica y para las investigaciones futuras.
\end{abstract}

Palabras clave: Mujeres maltratadas, trastorno de estrés postraumático, maltrato físico, maltrato psicológico.

\section{Physically battered women and psychologically battered women: a comparative study}

In this paper sociodemographic and psychopathological variables in a sample of 250 battered women who were under psychological treatment have been analysed. Likewise scores of both physical $(N=156)$ and psychological $(N=94)$ battered women were compared. The results showed a high level of anxiety - posttraumatic stress disorderand depression symptoms, as well as a poor adaptation to daily life activities. Differences between the two groups were not observed, in spite of a greater severity of behaviours implied in the group of physically battered women. Implications of this study for clinical practice and future research in this field are commented upon.

Key-words: Battered women, posttraumatic stress disorder, physical abuse, psychological abuse.

\section{INTRODUCCIÓN}

La violencia familiar representa un grave problema social, tanto por su alta incidencia en la población como por las

Correspondencia: Departamento de Personalidad, Evaluación y Tratamientos Psicológicos, Facultad de Psicología, Universidad del País Vasco, Avda. de Tolosa, 70, 20018 San Sebastián

Agradecimientos. Este estudio se ha financiado gracias a un convenio de investigación entre la Universidad del País Vasco, el Instituto Vasco de la Mujer. las Diputaciones de Vizcaya y Álava y el Ayuntamiento de Vitoria. El primer autor ha contado con una beca de investigación predoctoral del Gobierno Vasco (proyecto n. ${ }^{\circ}$ BFI96.080). consecuencias psicopatológicas que produce en las víctimas (Naciones Unidas, 1987). Actualmente el maltrato doméstico es un fenómeno epidémico que ha crecido a un ritmo más rápido incluso que los accidentes de coche, las agresiones sexuales y los robos. En realidad, la familia es el foco de violencia más destacado de nuestra sociedad. De hecho, según los estudios realizados en EEUU, entre el $15 \%$ y el $30 \%$ de las mujeres sufren algún tipo de agresión en la relación de pareja (Goldman, Horan, Warshaw, Kaplan y Hendricks-Matthews, 
1995; O'Leary y Arias, 1988; Stith, Williams y Rosen, 1992; Straus y Gelles, 1990).

En España los datos no son menos alarmantes. De hecho, según un informe reciente del Ministerio de Asuntos Sociales (2000), en nuestro país el $4 \%$ de la población femenina adulta (aproximadamente 650.000 mujeres) han sido maltratadas en el último año. Pero un aspecto destacable de este estudio es que hay un $12 \%$ adicional (aproximadamente $\mathbf{1 . 8 6 5 . 0 0 0}$ mujeres) que también son maltratadas y que, sin embargo, no tienen conciencia de serlo.

Este último aspecto es un reflejo de la existencia de unas cifras negras importantes. De hecho, en España hay alrededor de 27.000 denuncias anuales de maltrato en la pareja, pero tan sólo representan una mínima parte de los casos existentes (en torno al 10\%-30\% del total) (Torres y Espada, 1996). No debe extrañar que la dependencia económica y emocional de la mujer respecto al hombre, así como las circunstancias específicas de la mujer maltratada (baja autoestima, temor a perder los hijos, vergüenza, miedo al agresor, etc.), oculten parcialmente la realidad existente. En concreto, la consideración de que la violencia familiar atañe sólo al ámbito de lo privado y la relativa tolerancia social -al menos hasta ahorahan contribuido a inhibir la aparición del problema en sus justas dimensiones (Lorente, 2001).

La violencia en la pareja puede revestir diversas formas. Una de ellas es el maltrato físico, que ocurre cuando las conductas implicadas (puñetazos, golpes, patadas, amagos de estrangulamiento, etc.) son la expresión de un abuso físico. La situación de máximo riesgo para la integridad de la mujer puede situarse en el momento de la separación, cuando el agresor se da cuenta de que la pérdida es ya algo inevitable.
Otra manifestación de la violencia intrafamiliar, mucho menos estudiada, es el maltrato psicológico, que puede ser reflejo de diversas actitudes por parte del maltratador: hostilidad, que se presenta en forma de reproches, insultos y amenazas; desvalorización, que supone un desprecio de las opiniones, de las tareas o incluso del propio cuerpo de la víctima; e indiferencia, que representa una falta total de atención a las necesidades afectivas y a los estados de ánimo de la mujer.

No se debe confundir, sin embargo, la violencia psíquica con una mala relación de pareja, que se caracteriza por la desaparición del afecto, las broncas más o menos esporádicas y el deseo de poner fin a la relación. La violencia psíquica, por el contrario, es mucho más intensa y permanente, caracterizada por las conductas ya señaladas, y genera además consecuencias negativas clínicamente relevantes en la víctima.

Más específicamente, el maltrato doméstico está asociado a problemas psicopatológicos tales como el trastorno de estrés postraumático, la sintomatología depresiva, una baja autoestima, la ansiedad excesiva y, en general, un elevado grado de inadaptación a la vida cotidiana (Echeburúa, Corral, Amor, Sarasua y Zubizarreta, 1997a; Golding, 1999; McGruder-Johnson, Davidson, Gleavves, Stock y Finch, 2000).

Los principales objetivos de esta investigación han sido dos: en primer lugar, el análisis de las características sociodemográficas y psicopatológicas de una muestra amplia de mujeres maltratadas en busca de ayuda terapéutica; y en segundo lugar, el estudio comparativo de las víctimas de violencia física y de violencia psicológica en función de las características sociodemográficas y psicopatológicas, así como de las circunstancias del maltrato mismo. 


\section{MÉTODO}

\section{Sujetos}

La muestra de esta investigación consta de 250 mujeres víctimas de maltrato doméstico que acudieron en busca de tratamiento psicológico a diferentes Centros de Asistencia Psicológica para Víctimas de Violencia Familiar, ubicados en el País Vasco, entre los años 1995 y 1998.

En concreto, la muestra está formada por mujeres adultas que habían sido objeto de algún tipo de maltrato -psicológico o físico- por parte de su pareja (marido o novio). En algunos casos acudieron por iniciativa propia (medios de comunicación, folletos informativos, etc.); en otros, por alguna de las siguientes vías de derivación: Servicio de la Mujer; Ayuntamientos; Asistentes sociales de diferentes municipios; Policía; Otros profesionales; Teléfono nacional de asistencia al maltrato; Teléfono de la Esperanza; Conocidos y amigos, etc.

\section{Diseño}

Se trata de un estudio descriptivo que se ha llevado a cabo a partir de la evaluación de las víctimas antes del tratamiento (método de corte transversal). De este modo, se analizaron y compararon las frecuencias de las principales variables implicadas -sociodemográficas, de maltrato y psicopatológicas-en función del tipo de maltrato sufrido (físico o psicológico).

\section{Instrumentos de evaluación}

Los instrumentos de evaluación empleados en esta investigación han sido los siguientes:

a) Entrevista semiestructurada para las víctimas de maltrato doméstico
(Echeburúa y Corral, 1998). A partir de esta entrevista se evalúan las características sociodemográficas de las víctimas y la historia de victimización y de trastornos psicopatológicos, así como las circunstancias del maltrato doméstico. Un aspecto importante es que, al facilitar un clima de empatía, posibilita la expresión emocional de las víctimas y permite conocer la reacción del entorno familiar y social ante el trauma vivido.

b) Escala de Gravedad de Síntomas del Trastorno de Estrés Postraumático (EGS) (Echeburúa, Corral, Amor, Zubizarreta y Sarasua, 1997b). La Escala de Gravedad de Síntomas del Trastorno de Estrés Postraumático es una entrevista estructurada que sirve para evaluar la gravedad e intensidad de los síntomas de este cuadro clínico según los criterios diagnósticos del DSM-IV (American Psychiatric Association, 1994) - en víctimas de diferentes sucesos traumáticos. Esta escala, estructurada en un formato de tipo Likert de 0 a 3 según la frecuencia e intensidad de los síntomas, consta de 17 items, de los que 5 hacen referencia a los síntomas de reexperimentación, 7 a los de evitación y 5 a los de hiperactivación. El rango es de 0 a 51 en la escala global; de 0 a 15, en la subescala de reexperimentación; de 0 a 21 , en la de evitación; y de 0 a 15, en la de activación. La eficacia diagnóstica de la escala es muy alta $(95,45 \%)$ si se establece un punto de corte global de 15, y unos puntos de corte parciales de 5, 6 y 4 en las subescalas de reexperimentación, evitación e hiperactivación, respectivamente. Las propiedades psicométricas de la escala son asimismo muy satisfactorias (Echeburúa et al., 1997b). 
c) Inventario de Ansiedad Estado-Rasgo (STAI) (Spielberger, Gorsuch y Lushene, 1970) (versión española de TEA, 1982). El Inventario de Ansiedad Estado-Rasgo es un autoinforme que consta de 20 items relacionados con la ansiedad-rasgo y de otros 20 relacionados con la ansiedad-estado. El rango de las puntuaciones es de 0 a 60 en cada escala. El punto de corte para la población femenina en el STAI-Estado es de 31 (correspondiente al percentil 75). La fiabilidad test-retest es de 0,81 en la escala de ansiedad-rasgo, bastante más alta, como es lógico, que en la de ansiedad-estado $(r=0,40)$. La consistencia interna oscila de 0,83 a 0,92 . Por otra parte, la validez convergente con otras medidas de ansiedad oscila entre 0,58 y 0,79 .

d) Inventario de Depresión (BDI) (Beck, Rush, Shaw y Emery, 1979) (versión española de Vázquez y Sanz, 1997). Es un instrumento de autoinforme que consta de 21 items (rango: 0-63 puntos) y mide la intensidad de los síntomas depresivos. El punto de corte más utilizado para discriminar entre la población sana de la población aquejada de sintomatología depresiva es 18 (Steer y Beck, 1988). En sus items se da más importancia a los componentes cognitivos de la depresión que a los conductuales y somáticos. El coeficiente de fiabilidad por el método de las dos mitades es de 0,93. Desde la perspectiva de la validez convergente, la correlación con la evaluación clínica de la depresión oscila de 0,62 a 0,66 .

e) Escala de Valoración de la Depresión (HRS) (Hamilton, 1960) (versión española de Conde y Franch, 1984). Es una escala heteroaplicada que consta de 21 items (rango: 0-62 puntos) y que cuantifica la sintoma- tología depresiva de los pacientes, especialmente las alteraciones somáticas y conductuales. Suele ser empleada de forma complementaria al "Inventario de Deprcsión de Beck" (Beck et al., 1979). El punto de corte establecido para esta escala es de 18 puntos. Este instrumento posee una buena fiabilidad entre observadores $(r=0,90)$, tiene un buen nivel de validez concurrente con el $B D I(r=0,60)$ y es muy sensible a los cambios terapéuticos.

f) Escala de Autoestima (EAE) (Rosenberg, 1965). Es un instrumento de autoinforme que consta de 10 items (rango: 10-40 puntos) y que mide el grado de satisfacción que una persona tiene consigo misma. La mitad de los items están planteados de forma afirmativa y la otra mitad de forma negativa, con el objetivo de controlar la aquiescencia. Cuanto mayor es la puntuación, mayor es el grado de autoestima. El punto de corte en la población adulta, según el estudio de Ward (1977), es de 29. La versión española de esta escala se puede encontrar en Echeburúa y Corral (1998).

g) Escala de Inadaptación (IG) (Echeburúa y Corral, 1995). Es un autoinforme que consta de 6 items (rango: 0-30 puntos) y mide el grado en que el maltrato doméstico afecta a diferentes áreas de la vida cotidiana: trabajo, vida social, tiempo libre, relación de pareja y relación familiar. El punto de corte establecido es de 12 en la escala total y de 2 en cada uno de los items. Cuanto mayor es la puntuación, mayor es la inadaptación. En esta investigación se ha utilizado el ítem que refleja el grado de inadaptación global a la vida cotidiana, con un rango que oscila de 0 a 5 puntos. La prueba, así como la descripción de sus pro- 
piedades psicométricas de fiabilidad y validez, que han resultado ser satisfactorias, puede encontrarse en Echeburúa, Corral y FernándezMontalvo (2000).

\section{Procedimiento}

Las mujeres de esta investigación fueron evaluadas en el momento de su llegada inicial a los diferentes Centros de Asistencia Psicológica para Víctimas de Maltrato Doméstico ubicados en el País Vasco (Bilbao y Vitoria). La evaluación requirió aproximadamente dos horas y media y se distribuyó en dos sesiones, con un intervalo de una semana entre ellas. El protocolo de evaluación empleado, así como el orden de aplicación de las diferentes pruebas, fue el siguiente:

a) $1^{9}$ sesión: Recogida de datos de identificación personal; Entrevista Semiestructurada para las Víctimas de Maltrato Doméstico (Echeburúa, Corral, Sarasua, Zubizarreta y Sauca, 1994).

b) $2^{a}$ sesión: Escala de Gravedad de Síntomas del Trastorno de Estrés Postraumático (EGS) (Echeburúa et al., 1997b); Inventario de Ansiedad Estado/Rasgo (STAI) (Spielberger et al., 1970); Inventario de Depresión (BDI) (Beck et al., 1979); Escala de Valoración de la Depresión (HRS) (Hamilton, 1960); Escala de Autoestima (EAE) (Rosenberg, 1965); Escala de Inadaptación (IG) (Echeburúa y Corral, 1995).

El proceso completo de evaluación fue realizado por tres psicólogas clínicas, con formación en terapia de conducta y con más de 10 años de experiencia en el tratamiento de la violencia familiar. En la cumplimentación de los autoinformes una de las terapeutas siempre estuvo pre- sente para responder a todas las dudas que pudieran surgir en las pacientes.

La evaluación se insertaba en un contexto clínico en donde la persona encargada de evaluar a la víctima posteriormente se hacía cargo del tratamiento psicológico

\section{RESULTADOS}

A continuación se presentan los resultados referidos a las variables sociodemográficas, de maltrato y psicopatológicas correspondientes a las dos submuestras analizadas en este estudio (maltrato físico y maltrato psicológico). En concreto, el conjunto de las víctimas ( $\mathrm{N}=250)$ se ha dividido en dos grupos en funcibn del tipo de maltrato sufrido. En el primer grupo -maltrato físico $(n=156: 62 \%)-$ hay violencia física y psicologica; en el segundo -maltrato psicológico ( $n=94$ : $38 \%$ )-, exclusivamente violencia psicológica.

\section{a) Variables sociodemográficas}

Las características sociodemográficas de la muestra aparecen descritas en la Tabla 1. Se trata, en general, de personas que, aun siendo relativamente jóvenes (de menos de 40 años), cuentan con una larga historia de maltrato y que están casadas, pero que en casi la mitad de los casos están separadas o en trámites de separación. El nivel cultural y socioeconómico es más bien bajo. No suelen tener un trabajo extradoméstico; y si lo tienen, suele ser más bien poco cualificado.

Sólo una pequeña parte de las víctimas (en torno al $10 \%$ ) ha tenido una historia psiquiátrica anterior, referida sobre todo al ámbito de las alteraciones emocionales (ansiedad y depresión). Se trata, por tanto, de mujeres equilibradas mentalmente que sufren de trastornos psicológicos 
Tabla 1. Comparación de las variables sociodemográficas en función del tipo de maltrato

\begin{tabular}{|c|c|c|c|c|c|}
\hline & \multicolumn{2}{|c|}{$\begin{array}{l}\text { Maltrato físico } \\
\quad(\mathrm{N}=156)\end{array}$} & \multicolumn{2}{|c|}{$\begin{array}{l}\text { Maltrato psicológico } \\
(\mathrm{N}=94)\end{array}$} & \multirow[b]{2}{*}{$\chi^{2}$} \\
\hline & $\mathrm{N}$ & $(\%)$ & $\mathrm{N}$ & $(\%)$ & \\
\hline \multicolumn{6}{|l|}{ Estado civil } \\
\hline Casadas & 72 & $(46,2)$ & 51 & $(54,3)$ & 2,35 \\
\hline Solteras & $\mathbf{5}$ & $(3,2)$ & 2 & $(2,1)$ & (n.s.) \\
\hline Separadas & 21 & $(13,5)$ & 14 & $(14,9)$ & \\
\hline En trámites separación & 58 & $(37,2)$ & 27 & $(28,7)$ & \\
\hline \multicolumn{6}{|l|}{ Nivel económico } \\
\hline Bajo & 48 & $(30,8)$ & 25 & $(26,6)$ & 5,78 \\
\hline Medio-Bajo & 37 & $(23,7)$ & 21 & $(22,3)$ & (n.s.) \\
\hline Medio & 52 & $(33,3)$ & 33 & $(35,1)$ & \\
\hline Medio-Alto & 7 & $(4,5)$ & 11 & $(11,7)$ & \\
\hline Alto & 12 & $(7,7)$ & 4 & $(4,3)$ & \\
\hline \multicolumn{6}{|l|}{ Nivel cultural } \\
\hline Sin estudios & 37 & $(23,7)$ & 16 & (17) & 1,58 \\
\hline Estudios primarios & 71 & $(45,5)$ & 46 & $(48,9)$ & (n.s.) \\
\hline Estudios secundarios & 39 & $(25)$ & 26 & $(27,7)$ & \\
\hline Estudios universitarios & 9 & $(5,8)$ & 6 & $(6,4)$ & \\
\hline \multicolumn{6}{|l|}{ Profesión } \\
\hline Estudiante & 4 & $(2,6)$ & 2 & $(2,1)$ & 3,25 \\
\hline Ama de casa & 48 & $(30,8)$ & 25 & $(26,6)$ & (n.s.) \\
\hline Servicio doméstico & 21 & $(13,5)$ & 17 & $(18,1)$ & \\
\hline Otra profesión & 57 & $(36,5)$ & 40 & $(42,6)$ & \\
\hline En paro & 26 & $(16,7)$ & 10 & $(10,6)$ & \\
\hline \multicolumn{6}{|l|}{$\mathrm{H}^{\otimes}$ psiquiátrica anterior } \\
\hline sí & 21 & $(13,5)$ & 7 & $(7,4)$ & 2,13 \\
\hline NO & 135 & $(86,5)$ & 87 & $(92,6)$ & (n.s.) \\
\hline \multicolumn{6}{|l|}{ Apoyo social $(\mathrm{N}=212)$} \\
\hline sf & 69 & $(50,4)$ & 41 & $(54,7)$ & 0,36 \\
\hline NO & 68 & $(49,6)$ & 34 & $(45,3)$ & (n.s.) \\
\hline \multicolumn{6}{|l|}{ Apoyo familiar $(\mathrm{N}=212)$} \\
\hline Sí & 91 & $(66,4)$ & 55 & $(73,3)$ & 1,08 \\
\hline \multirow[t]{2}{*}{ NO } & 46 & $(33,6)$ & 20 & $(26,7)$ & (n.s.) \\
\hline & Media & DT & Media & DT & $t$ \\
\hline \multicolumn{6}{|l|}{ Edad media } \\
\hline (Rango: 19-66 años) & 38,36 & 10,02 & 38,50 & 8,31 & $-0,12$ (n.s.) \\
\hline \multicolumn{6}{|l|}{ Número de hijos } \\
\hline (Rango: 0-6) & 1,91 & 1,26 & 1,74 & 1,13 & 1,05 (n.s.) \\
\hline
\end{tabular}

Nota: (n.s.) no significativo

actualmente como consecuencia de una situación de maltrato crónico.

No deja de ser significativo que un porcentaje considerable de estas víctimas carezca de redes de apoyo. En concreto, un tercio de ellas no cuenta con apoyo familiar y casi la mitad carece de apoyo social.

Por lo que se refiere en concreto a la comparacion entre los grupos, no hay diferencias significativas en ninguna de las variables estudiadas.

\section{b) Circunstancias del maltrato}

En cuanto a la duración del problema, un primer dato es que la situación de maltrato es más bien crónica y de larga evolución. Al margen de que el rango es 
muy amplio, la duración media del maltrato es de más de 10 años, mayor aún en las víctimas de maltrato físico $(t=2,62 ; p<0,05)$. Un segundo dato es que el maltrato es de instauración pre$\mathrm{coz}$ en la relación de pareja, pero con ciertas diferencias: la violencia comienza dentro de los dos primeros años de convivencia (noviazgo o primer año de convivencia en pareja) en el $82 \%$ de los casos de maltrato físico, pero en menor proporción en las víctimas de maltrato psicológico (59\%). Asimismo, en casi todas las víctimas, los últimos episodios violentos han sido muy recientes: más del $70 \%$ de las mujeres han sido maltratadas en los últimos 3 meses. La significación clínica de las diferencias entre uno y otro grupo en esta variable no está muy clara (Tabla 2).

Tabla 2. Comparación de las circunstnacias del maltrato en función del tipo de violencia ejercida

\begin{tabular}{|c|c|c|c|c|c|}
\hline & \multicolumn{2}{|c|}{$\begin{array}{l}\text { Maltrato físico } \\
\qquad(N=156)\end{array}$} & \multicolumn{2}{|c|}{$\begin{array}{l}\text { Maltrato psicologico } \\
(N=94)\end{array}$} & \multirow[b]{2}{*}{$\chi^{2}$} \\
\hline & $\mathrm{N}$ & $(\%)$ & $\mathrm{N}$ & $(\%)$ & \\
\hline \multicolumn{6}{|l|}{ Primer episodio de maltrato } \\
\hline Noviazgo & 39 & $(25,0)$ & 13 & $(13,8)$ & $19,78 \star \star \star$ \\
\hline Primer año matrimonio & 89 & $(57,1)$ & 43 & $(45,7)$ & \\
\hline 2-5 años de matrimonio & 19 & $(12,2)$ & 17 & $(18,1)$ & \\
\hline Más de 5 años matrimonio & 9 & $(5,8)$ & 21 & $(22,3)$ & \\
\hline \multicolumn{6}{|l|}{ Último episodio de maltrato } \\
\hline $0-1$ mes & 90 & $(57,7)$ & 54 & $(57,4)$ & $7,94 *$ \\
\hline 1-3 meses & 38 & $(24,4)$ & 12 & $(12,8)$ & \\
\hline 3-6 meses & 11 & $(7,1)$ & 9 & $(9,6)$ & \\
\hline Más de 6 meses & 17 & $(10,9)$ & 19 & $(20,2)$ & \\
\hline \multicolumn{6}{|l|}{ Maltrato en familia de origen } \\
\hline sf & 38 & $(24,4)$ & 13 & $(13,8)$ & 4,00 * \\
\hline NO & 118 & $(75,6)$ & 81 & $(86,2)$ & \\
\hline \multicolumn{6}{|l|}{ Relación sexual forzada } \\
\hline sf & 80 & $(51,3)$ & 13 & $(13,8)$ & $35,22 * \star *$ \\
\hline NO & 76 & $(48,7)$ & 81 & $(86,2)$ & \\
\hline \multicolumn{6}{|l|}{ Denuncia } \\
\hline Sf & 95 & $(60,9)$ & 15 & (16) & $48,08 \star \star \star$ \\
\hline NO & 61 & $(39,1)$ & 79 & (84) & \\
\hline \multicolumn{6}{|l|}{ Salida del hogar } \\
\hline sf & 88 & $(56,4)$ & 16 & (17) & $37,46 * \star \star$ \\
\hline NO & 68 & $(43,6)$ & 78 & (83) & \\
\hline \multicolumn{6}{|l|}{ Piso de acogida } \\
\hline sf & 22 & $(14,1)$ & 3 & $(3,2)$ & $7,76 * *$ \\
\hline NO & 134 & $(85,9)$ & 91 & $(96,8)$ & \\
\hline \multicolumn{6}{|l|}{ Maltrato durante el embarazo } \\
\hline Sí & 75 & $(54.7)$ & 19 & $(23,2)$ & $20,87 \star \star \star$ \\
\hline NO & 62 & $(45,3)$ & 63 & $(76,8)$ & \\
\hline \multicolumn{6}{|l|}{ Maltrato a los hijos } \\
\hline sí & 96 & $(70,1)$ & 41 & $(50)$ & $8,82 * *$ \\
\hline NO & 41 & $(29,9)$ & 41 & (50) & \\
\hline \multicolumn{6}{|c|}{ Tipo de maltrato sufrido por los hijos } \\
\hline Maltrato físico & 42 & $(43,8)$ & 8 & $(19,5)$ & \\
\hline \multirow[t]{2}{*}{ Maltrato psicológico } & 52 & $(56,3)$ & 33 & $(80,5)$ & $7,28 \star \star$ \\
\hline & Media & DT & Media & DT & $t$ \\
\hline $\begin{array}{l}\text { Años de maltrato } \\
\text { (Rango: } 1-40 \text { años) }\end{array}$ & 13,96 & 9,59 & 10,97 & 8,19 & $t=2,62 * *$ \\
\hline
\end{tabular}

Nota: ${ }^{\star} p<0,05 ;{ }^{\star \star} p<0,01 ;{ }^{\star \star \star} p<0,001$ 
Desde la perspectiva de los antecedentes familiares, sólo una pequeña parte de la muestra -el $20 \%$ - ha sufrido o ha sido testigo de maltrato en la familia de origen, pero es significativamente mayor en las víctimas de maltrato físico $\left(\chi^{2}=4,00 ; p<0,05\right)$.

La intensidad de la violencia familiar es mayor en las víctimas de maltrato físico. Así, por ejemplo, las relaciones sexuales forzadas, el maltrato durante los embarazos, las denuncias, las salidas del hogar y el recurso a los pisos de acogida son mucho más frecuentes en las víctimas de violencia física que en las de maltrato psicológico.

Por otra parte, la violencia contra la mujer tiende a hacerse extensiva a los hijos, pero con más frecuencia en el caso de los maltratadores físicos $\left(\chi^{2}=8,82\right.$; $p<0,05)$, con una modalidad de maltrato similar a la que se ejerce contra la mujer.

\section{c) Características psicopatológicas}

A continuación se describe el perfil psicopatológico de las víctimas estudiadas en relación con el trastorno de estrés postraumático y con otras características clínicas (Tabla 3).

\section{Trastorno de estrés postraumático}

La tasa de prevalencia del trastorno de estrés postraumático en la muestra total estudiada es alta. Un $46 \%$ del total de la muestra está afectada por este cuadro clí-

Tabla 3. Comparaciones de medias entre diferentes variables psicopatológicas, en función del tipo de maltrato sufrido por la mujer

\begin{tabular}{llll}
\hline \multirow{2}{*}{ ESCALAS } & \multicolumn{2}{c}{$\begin{array}{c}\text { Maltrato físico } \\
(\mathrm{N}=137)\end{array}$} & \multicolumn{2}{c}{$\begin{array}{c}\text { Maltrato psicológico } \\
(\mathrm{N}=75)\end{array}$} \\
& Media $^{\text {(DT) }}$ & Media $^{\text {(DT) }}$ & \\
\hline
\end{tabular}

TRASTORNO DE ESTRÉS POSTRAUMÁTICO

$\begin{array}{cccccc}\begin{array}{c}\text { ESCALA GLOBAL } \\ \text { (Rango: } 0-51)\end{array} & 20,31 & (8,97) & 20,20 & (9,23) & \begin{array}{c}0,08 \\ \text { (n.s.) }\end{array}\end{array}$

ESCALAS ESPECÍFICAS

$\begin{array}{lllllc}\begin{array}{l}\text { Reexperimentación } \\ \quad \text { (Rango: } 0-15)\end{array} & 6,14 & (2,95) & 5,80 & (3,20) & \begin{array}{c}0,77 \\ \text { (n.s.) }\end{array} \\ \begin{array}{l}\text { Evitación } \\ \quad \text { (Rango: } 0-21)\end{array} & 6,58 & (3,69) & 7,16 & (4,09) & -1,04 \\ \begin{array}{l}\text { Aumento de la Activación } \\ \quad \text { (Rango: } 0-15)\end{array} & 7,70 & (4,38) & 7,20 & (4,14) & 0,81 \\ \text { (n.s.) } & & & & \text { (n.s.) }\end{array}$

OTROS SÍNTOMAS PSICOPATOLÓGICOS Y DE INADAPTACIÓN

\begin{tabular}{cccccc}
$\begin{array}{c}\text { Ansiedad (STAI-E) } \\
\text { (Rango: } 0-60)\end{array}$ & 40,79 & $(11,59)$ & 41,01 & $(11,51)$ & $\begin{array}{c}-0,14 \\
\text { (n.s.) }\end{array}$ \\
$\begin{array}{c}\text { Depresión }(B D) \\
\text { (Rango: } 0-63)\end{array}$ & 18,74 & $(9,73)$ & 20,11 & $(9,52)$ & $\begin{array}{c}-0,99 \\
\text { (n.s.) }\end{array}$ \\
$\begin{array}{c}\text { Depresión }(H R S) \\
\text { (Rango: } 0-62)\end{array}$ & 23,43 & $(12,13)$ & 24,71 & $(12,26)$ & $-0,73$ \\
$\begin{array}{c}\text { Autoestima (EAE) } \\
\text { (Rango: } 10-40)\end{array}$ & 27,46 & $(6,0)$ & 27,51 & $(5,61)$ & $-0,06$ \\
$\begin{array}{c}\text { Inadaptación global } \\
\text { (Rango: } 0-5)\end{array}$ & 3,99 & $(1,28)$ & 4,04 & $(1,12)$ & $-0,31$ \\
\hline
\end{tabular}

Nota: (n.s.) no significativo 
nico, pero hay un $16 \%$ adicional que presentan el denominado por Hickling y Blanchard (1992) subsíndrome de estrés postraumático (es decir, dos de los tres criterios diagnósticos: reexperimentación más evitación o hiperactivación). Por tanto, sólo hay un $\mathbf{3 8 \%}$ de mujeres maltratadas que no presenta sintomatología clínicamente relevante de este trastorno. Por otra parte, al comparar la tasa de prevalencia del trastorno de estrés postraumático en función del tipo de maltrato sufrido (físico/psicológico), no se encontraron apenas diferencias. En concreto, en ambos grupos la prevalencia de este cuadro clínico es del 46\%; y la del subsíndrome, en las víctimas de maltrato físico es del $18 \%$, y en las de violencia psicológica del $11 \%\left(\chi^{2}=2,39\right.$; n.s. $)$.

Desde una perspectiva dimensional, la intensidad global del trastorno de estrés postraumático en las víctimas de violencia familiar es más bien alta. La puntuación media obtenida en la escala global y en cada subescala de este cuadro clínico supera todos los puntos de corte establecidos en la validación de este instrumento de medida. Tampoco en este caso hay diferencias entre los grupos.

\section{Otras variables psicopatológicas y de inadaptación}

En general, las víctimas de maltrato estudiadas presentan un nivel muy elevado de síntomas psicopatológicos. Así, por ejemplo, en cuanto a la ansiedad, el $83 \%$ de la muestra total supera el percentil 75. Por lo que se refiere a la depresión, nada menos que el $50,5 \%$ de las mujeres en el $B D I$ y el $67,5 \%$ en el HRS superan los puntos de corte establecidos: de este modo, entre la mitad y los dos tercios de las mujeres de la muestra están aquejadas de una depresión media o grave. Asimismo las víctimas muestran un grado de autoestima más bien bajo. De hecho, el $56 \%$ de ellas están por debajo del punto de corte de la población normal.

Por lo que se refiere a la adaptación a la vida cotidiana, el $71 \%$ de las víctimas presenta un nivel muy alto de inadaptación. De este modo, el maltrato interfiere claramente en el funcionamiento diario de estas personas.

En cuanto a la comparación entre los grupos, no hay diferencias significativas en ninguna de las variables estudiadas.

\section{CONCLUSIONES}

En esta investigación se ha estudiado a una muestra de mujeres maltratadas que acudieron en busca de tratamiento psicológico. La muestra de este estudio cuenta con la ventaja de ser muy amplia, pero tiene el inconveniente de no recoger a las víctimas ocultas que no buscan ayuda terapéutica y de no abarcar a todo el espectro socioeconómico. Al ser víctimas que han acudido a un tratamiento gratuito en los Centros de Asistencia Psicológica para Víctimas de Violencia Familiar, cabe pensar que no estén representadas suficientemente las mujeres maltratadas de clases sociales más altas, que pueden utilizar otro tipo de recursos privados.

Un dato de interés es que más de la tercera parte de las víctimas (en concreto, el $38 \%$ ) acuden a la consulta por un problema de maltrato exclusivamente psicológico. Por tanto, lo que genera un problema psicológico y una petición de ayuda terapéutica no se puede reducir exclusivamente a la violencia física. El maltrato psicológico puede ser un primer peldaño en la escalada de la violencia física, pero en muchos casos tiene entidad en sí mismo, sin traspasar los límites de otro tipo de violencia.

La violencia familiar es crónica y se instaura tempranamente en la relación de pareja, por lo que se trata de un fenóme- 
no de larga evolución. La búsqueda de ayuda terapéutica en la muestra estudiada tiene lugar más de 10 años después de surgido el maltrato. La dependencia económica, social y afectiva de la mujer respecto al varón, así como las expectativas ingenuas de una remisión espontánea de la violencia, explican estas consultas tardías (Lorente, 2001).

La separación puede ser una condición necesaria, pero no suficiente, para resolver los problemas de maltrato. No deja de ser significativo que, a pesar de que la mitad de las mujeres están en trámites de separación o llevan separadas más de tres meses del agresor, casi el $58 \%$ de la muestra ha sido maltratada en el transcurso del último mes. Quiere ello decir que, a pesar de que la mujer se aleje del agresor, puede entrar en una nueva fase de acoso, sufrimiento e indefensión (Garrido, 2001; Sarasua y Zubizarreta, 2000).

La ausencia de apoyo social en casi la mitad de la muestra es un aspecto significativo. Esta situación está favorecida por la presión del agresor, así como por el miedo y la vergüenza social sufridos por la víctima (en concreto, el temor al qué dirán). Este contexto genera una mayor dependencia del agresor, quien, a su vez, experimenta un aumento del dominio a medida que se percata del mayor aislamiento de la víctima (Rojas Marcos, 1995).

Un hallazgo importante de esta investigación es que tan sólo el $10 \%$ de las mujeres estudiadas tenía una historia psiquiátrica anterior. De esta forma, quedan en entredicho las teorías explicativas que ponen énfasis en que el origen del maltrato es responsabilidad de la mujer, bien porque ésta presenta ciertos desequilibrios emocionales (Gayford, 1975), bien porque tiene una necesidad de sufrir por naturaleza (Faulk, 1974) o porque es constitutivamente dependiente $y$, por tanto, más vulnerable a la violencia.
El maltrato a la mujer tiende a formar parte de un estilo de relación violento por parte del agresor. De hecho, el maltrato se extiende a los hijos en más de la mitad de los casos de este estudio. Es más, como se ha puesto de relieve en algunas investigaciones (Fernández-Montalvo y Echeburúa, 1997; HoltzworthMunroe y Stuart, 1994), aproximadamente uno de cada cuatro agresores se comportan de forma violenta tanto dentro como fuera del hogar.

Por otra parte, la violencia familiar, en lo que respecta a las víctimas, no se articula necesariamente en una cadena de transmisión generacional. En concreto, el $80 \%$ de la muestra no había sufrido ni observado maltrato en su familia de origen. $\mathrm{Al}$ margen de que, probablemente, es más fácil aprender a ser agresor que a ser víctima, en la violencia familiar los antecedentes inmediatos de la vida adulta (situaciones de estrés, dificultades de comunicación, problemas en la resolución de problemas, etc.) desempeñan un papel más importante que los antecedentes remotos de la niñez (Echeburúa y Corral, 1998).

Las víctimas de violencia familiar presentan un perfil psicopatológico preciso, caracterizado por el trastorno de estrés postraumático y por síntomas de ansiedad y de depresión, así como de baja autoestima. El resultado de todos estos síntomas clínicos es una profunda inadaptación a la vida diaria y una interferencia grave en el funcionamiento cotidiano. El perfil obtenido en este estudio resulta congruente con los datos obtenidos en otras investigaciones (Echeburúa et al., 1997a; Golding, 1999).

En cuanto a las diferencias entre los grupos de maltrato físico y de maltrato psicológico, las variables demográficas son similares, pero las circunstancias de la violencia son distintas en uno y otro caso. En concreto, en el grupo de maltrato físico son mayores la gravedad 
(comienzo anterior del maltrato, violencia de más larga evolución, extensión de la misma más frecuente a los hijos, etc.) y la intensidad (presencia de relaciones sexuales forzadas, maltrato durante los embarazos, presentación de denuncias, salidas del hogar, etc.). Respecto a los antecedentes familiares de violencia en el hogar, son, en general, relativamente poco frecuentes (un 20\%), pero están más presentes en el grupo de maltrato físico.

Sin embargo, y a pesar de una mayor gravedad objetiva de la violencia en el grupo de maltrato físico, no aparecen diferencias significativas en el perfil psicopatológico entre las víctimas de uno y otro grupo, como ha ocurrido también en otros estudios (Echeburúa et al., 1997a). La discrepancia entre la gravedad objetiva y la subjetiva puede dar cuenta de estos resultados. Es decir, las víctimas de maltrato psicológico están en una situación de menor gravedad, pero se sienten igual de mal que las víctimas de violencia física. Las humillaciones continuas tienen un impacto sobre la estabilidad emocional de las víctimas similar al producido por las agresiones físicas (Follingstad, Rutledge, Serg, House y Ploek, 1990).

Por ello, una línea de investigación futura de gran interés es la evaluación del daño psicológico en las víctimas de maltrato y de delitos violentos. Conocer con detalle el alcance de las lesiones psíquicas y de las secuelas emocionales va a permitir detectarlas tempranamente e intervenir adecuadamente sobre ellas.

\section{REFERENCIAS BIBLIOGRÁFICAS}

American Psychiatric Association (1994). Diagnostic and statistical manual of mental disorders ( $4^{\circ}$. ed.). Washington, DC: APA. Beck, A.T., Rush, A.J., Shaw, B.F., y Emery, G. (1979). Cognitive therapy of depression. New York: Guilford Press.
Conde, V., y Franch, J.I. (1984). Escalas de evaluación comportamental para la cuantificación de la sintomatología psicopatológica en los trastornos angustiosos $y$ depresivos. Madrid: Laboratorios Upjohn.

Echeburúa, E., y Corral, P. (1995). Trastorno de estrés postraumático. En A. Belloch, B. Sandín y F. Ramos (Eds.), Manual de psicopatología (Vol. 2) (pp. 171-186). Madrid: McGraw-Hill.

Echeburúa, E., y Corral, P. (1998). Manual de violencia familiar. Madrid: Siglo XXI.

Echeburúa, E., Corral, P. Amor, P.J., Sarasua, B., y Zubizarreta, I. (1997a). Repercusiones psicopatológicas de la violencia doméstica en la mujer: un estudio descriptivo. Revista de Psicopatología y Psicología Clínica, 2, 7-19.

Echeburúa, E., Corral, P., Amor, P.J., Zubizarreta, I., y Sarasua, B. (1997b). Escala de Gravedad de Síntomas del Trastorno de Estrés Postraumático: propiedades psicométricas. Análisis y Modificación de Conducta, 23, 503-526.

Echeburúa, E., Corral, P., y Fernández-Montalvo, J. (2000). Escala de Inadaptación: propiedades psicométricas en contextos clínicos. Análisis y Modificación de Conducta, 26, 325-340.

Faulk, M. (1974). Men who assault their wives. Medicine, Science and the Law, 14, 180-183.

Fernández-Montalvo, J., y Echeburúa, E. (1997). Variables psicopatológicas y distorsiones cognitivas de los maltratadores en el hogar: un análisis descriptivo. Análisis y Modificación de Conducta, 23, 151180.

Follingstad, D., Rutledge, L., Serg, B., House, E., y Ploek, D. (1990). The role of emotional abuse in physical abusive relationships. Journal of Family Violence, 5, 107120.

Garrido, V. (2001). Amores que matan. Valencia: Algar.

Gayford, J.J. (1975). Wife battering: a preliminary survey of 100 cases. British Medical Journal, 30, 194-197.

Golding, J.M. (1999). Intimate partner violence as a risk factor for mental disorders: A meta-analysis. Journal of Family Violence 14, 99-132. 
Goldman, L.S., Horan, D., Warshaw, C., Kaplan, S., y Hendricks-Matthews, M.B. (1995). Diagnostic and treatment guidelines on mental health effects of family violence. American Medical Association: Chicago.

Hamilton, M. (1960). A rating scale for depression. Journal of Neurology, Neurosurgery and Psychiatry, 23, 53-62.

Holtzworth-Munroe, A., y Stuart, G.L. (1994). Typologies of male batterers: Three subtypes and the differences among them. Psychological Bulletin, 116, 476-497.

Lorente, M. (2001). Mi marido me pega lo normal. Barcelona: Crítica.

McGruder-Johnson, A.K., Davidson, E.S., Gleavves, D.H., Stock, W., y Finch, J.F. (2000). Interpersonal violence and posttraumatic symptomatology. The effects of ethnicity, gender, and exposure to violent events. Journal of Interpersonal Violence, 15, 205221.

Ministerio de Trabajo y Asuntos Sociales, e Instituto de la Mujer (2000). Macroencuesta. Ministerio de Trabajo y Asuntos Sociales.

Naciones Unidas (1987). Violence against women in the family. New York.

O'Leary, K.D., y Arias, I. (1988). Prevalence, correlates and development of spouse abuse. En R.D. Peters y R.J. McMahon. (Eds.). Social learning in system approaches to marriage and the family. New York: Brunner/Mazel.

Rojas Marcos, L. (1995). Las semillas de la violencia. Madrid: Espasa-Calpe.

Rosenberg, M. (1965). Society and the Ado- lescent Self Image. Princeton: Printeton University Press.

Sarasua, B., y Zubizarreta, I. (2000). Violencia en la pareja. Málaga: Aljibe.

Spielberger, C.D., Gorsuch, R.L., y Lushene, R.E. (1970). Manual for the State/Trait Anxiety Inventory. Palo Alto, CA. Consulting Psychologist Press (versión española, TEA, 1982).

Steer, R.A., y Beck, A.T. (1988). Beck Depression Inventory. En M. Hersen y A.S. Bellack (Eds.), Dictionnary of Behavioral Assessment Techniques. Nueva York: Pergamon Press.

Stith, S., Williams, M., y Rosen, K. (1992). Psicosociología de la violencia en el hogar. Bilbao: Desclée de Brouwer (original, 1990).

Straus, M.A., y Gelles, R.J. (1990). How violent are american families? Estimates from the National Family Violence Resurvey and other studies. En M.A. Straus y R.J. Gelles (Eds.). Physical Violence in American Families. New Brunswick, NJ: Transaction Publishers.

Torres, P., y Espada, F.J. (1996). Violencia en casa. Madrid: Aguilar.

Vázquez, C., y Sanz, J. (1997). Fiabilidad y valores normativos de la versión española del Inventario para la Depresión de Beck de 1978/Reliability and norm data of the Spanish version of the 1978 Beck Depression Inventory. Clínica y Salud, 8, 403-422.

Ward, R.A. (1977). Aging group consciousness. Implications in a older sample. Sociology and Social Research, 61, 496519. 